\title{
The JUNO Calibration Strategy and Simulation
}

\author{
Kangfu Zhu' ${ }^{1}$, Qingmin Zhang ${ }^{2}$ on behalf of the JUNO collaboration \\ Department of Nuclear Science and Technology, School of Energy and Power Engineering, Xi'an \\ Jiaotong University, Xi'an 710049, China
}

\begin{abstract}
The Jiangmen Underground Neutrino Observatory (JUNO) is designed to primarily measure the neutrino Mass Ordering (MO) by detecting reactor anti-neutrinos via inverse beta decay. JUNO also has other rich physical potentials. Its Central Detector (CD), which is an acrylic sphere with a diameter of $35.4 \mathrm{~m}$, filled by approximately $20 \mathrm{kton}$ of liquid scintillator (LS), is equipped with large photomultipliers (18k for the CD $+2 \mathrm{k}$ for the Water Pool) and small photomultipliers $(25,600)$ to measure the energy resolution of neutrinos with an unprecedented energy resolution of $3 \% / \sqrt{E}$ and an energy non-linearity better than $1 \%$. Accordingly, a calibration complex, including Automatic Calibration Unit (ACU), Cable Loop System (CLS), Guide Tube Calibration System (GTCS) and Remotely Operated under-liquid-scintillator Vehicles (ROV), is designed to deliver multiple radioactive sources for the energy coverage of reactor neutrinos and $\mathrm{CD}$ full-volume. In this proceeding, the new design details and up-to-date progress about JUNO calibration system are presented.
\end{abstract}

40th International Conference on High Energy Physics - ICHEP2020

July 28 - August 6, 2020

Prague, Czech Republic (virtual meeting)

${ }^{1}$ Speaker, E-mail: zhukangfu@stu.xjtu.edu.cn

${ }^{2}$ Corresponding author,E-mail: zhangqingmin@mail.xjtu.edu.cn

(c) Copyright owned by the author(s) under the terms of the Creative Commons 


\section{Introduction of JUNO Energy Response}

The Jiangmen Underground Neutrino Observatory (JUNO), which will be constructed at Kaiping, Jiangmen in South China, is designed to determine the neutrino mass ordering by detecting reactor anti-neutrinos, with an expected precision of $3 \sigma$ reached in 6 years of data taking [1]. Its Central Detector (CD), which is an acrylic sphere with a diameter of $35.4 \mathrm{~m}$, filled by approximately 20 kton of liquid scintillator (LS), is equipped with large photomultipliers (PMTs, 18k for the CD $+2 \mathrm{k}$ for the Water Pool) and small PMTs $(25,600)$ to measure the energy resolution of neutrinos with an unprecedented energy resolution of $3 \% / \sqrt{\mathrm{E}}$ and an energy non-linearity better than $1 \%$ [2]. JUNO energy response is strongly position-dependent due to the detector's complicated structure and large dimension, so the calibration system is very critical and has been designed [3].

\section{JUNO Calibration System and its Calibration Strategy}

The calibration system has been designed for full volume coverage, as shown in Fig.1. It uses many radioactive sources for the energy coverage of reactor neutrinos.
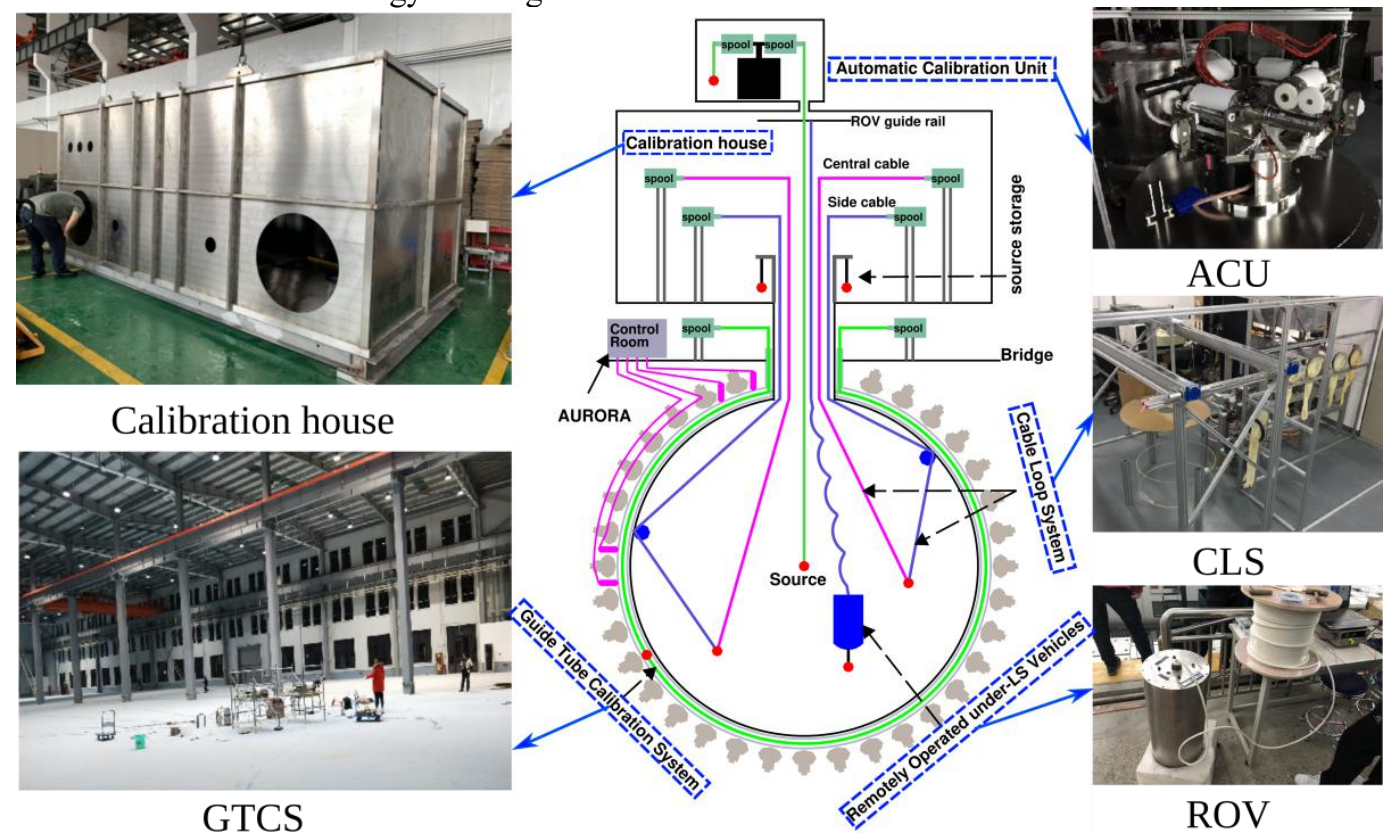

Figure 1: Scheme of JUNO calibration system.

\section{JUNO Calibration Simulation}

In the calibration simulation, we used 3 calibration systems to deliver different sources to specified positions to perform non-uniformity and non-linearity corrections. Finally, we combined the data from them to get an accurate energy measurement [4] .

\subsection{Non-uniformity Correction}

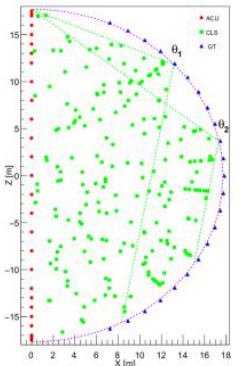

(a)

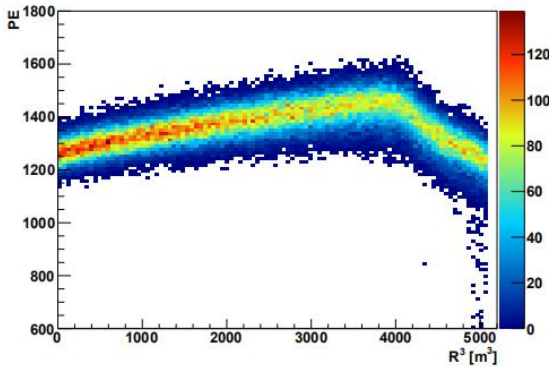

(b)

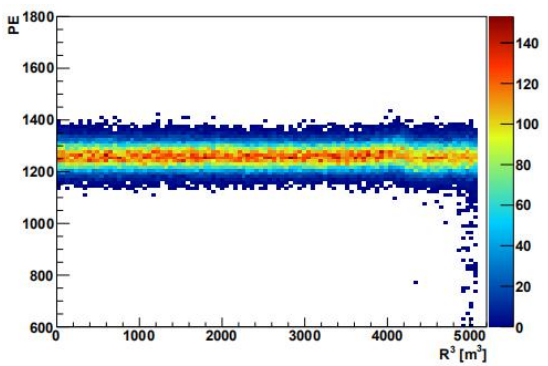

(c)

Figure 2: (a) 250 calibration points obtained with Am-C source from ACU/CLS/GTCS. (b) Energy resolution, displayed in photoelectrons (PEs), before correction is 7\%. (c) Energy resolution after correction reaches $3.02 \%$. 
JUNO response function can be calibrated by using the data from the given calibration points with ACU, CLS and GTCS (or ROV), see Fig. 2(a). A simple spline function is used to predict the "blank" region and the energy response uniformity is corrected with the correction function obtained from the calibration [5]. As shown in Fig. 2, an effective energy resolution of 3.02\% has been achieved after correction [6].

\subsection{Non-linearity Correction}

Energy linearity is corrected by placing various sources at CD center with ACU. Eight different gamma sources, specified in Fig. 3(a)[6], are used to study the detector's linearity. As a verification test, the energy bias (non-linearity) to mono-energetic electron positioned at CD center is simulated, which is found to be $<1 \%$ after non-linearity correction, see Fig.3(b) [7].

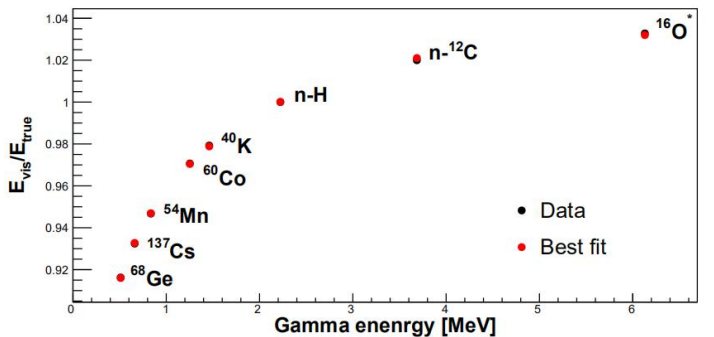

(a)

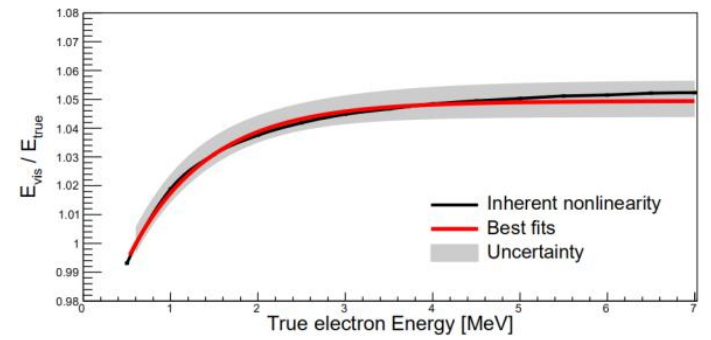

(b)

Figure 3: (a) Gamma non-linearity. (b) Electron non-linearity.

\subsection{Overall Energy Resolution}

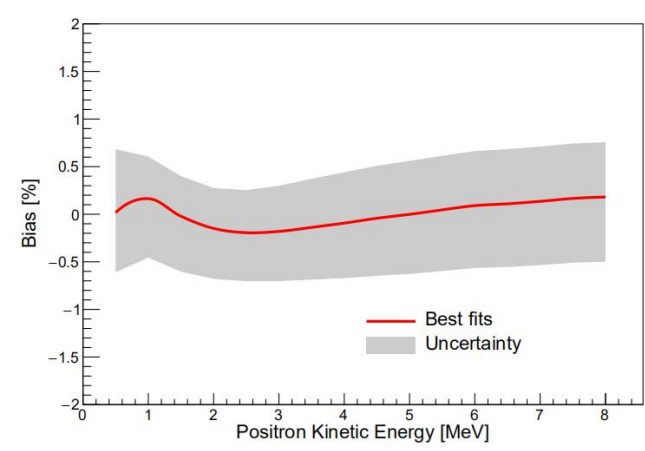

(a)

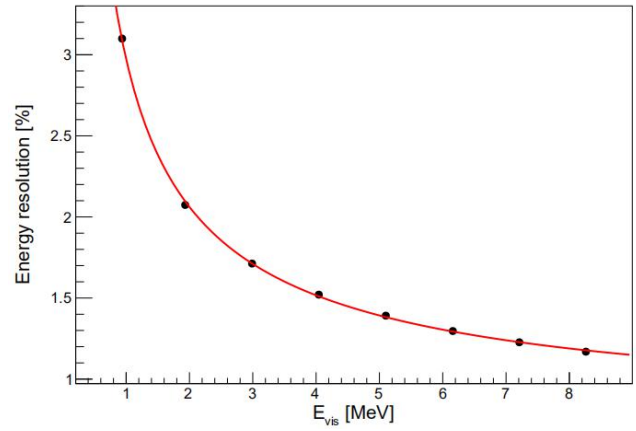

(b)

Figure 4: (a) Mean bias after correction. (b) Energy resolution after correction.

The simulated mono-energetic positron events are uniformly distributed in $\mathrm{CD}$ and the non-uniformity correction is applied. As shown in Fig.4 [6], the bias is less than $0.1 \%$ and the energy resolution is approximately $3.0 \%$ [8].

\section{References}

[1] J. Cao, Daya Bay and Jiangmen Underground Neutrino Observatory (JUNO) neutrino experim ents (in Chinese)[J]. Sci Sin-Phys Mech Astron, 2014, 44:1025-1040, doi: 10.1360/SSPMA201 4-00174.

[2] Y. K. Heng, Towards a comprehensive neutrino program: the design and progress of JUNO [R], APS April Meeting, Columbus, 2018.

[3] F. Y. Zhang, Consideration of Calibration Plan [R], Beijing: IHEP, 2019.

[4] Q. M. Zhang et al., The JUNO Calibration System [J], 39 ${ }^{\text {th }}$ International Conference on Hig h Energy Physics, Seoul, Korea,4-11 July, 2018, Proceedings of Science, PoS(ICHEP2018).

[5] F. Y. Zhang, Calibration for energy non-linearity and resolution [R], Beijing: IHEP, 2018.

[6] F. Y. Zhang et al,Calibration strategy of the JUNO experiment[J].10.1007/JHEP03(2021)004, ID, 10.1007/JHEP03(2021)004.

[7] F. Y. Zhang, Consideration of Calibration Plan [R], Beijing: IHEP, 2019.

[8] F. Y. Zhang, Calibration Strategy of the JUNO Experiment [R], Beijing: IHEP, 2020. 